\title{
HIDRODESSULFURIZAÇÃO DE DIBENZOTIOFENO E 4,6-DIMETILDIBENZOTIOFENO EM CATALISADOR $\mathrm{NiMoP} / \mathrm{Al}_{2} \mathrm{O}_{3}$
}

\author{
M. D. MELLO ${ }^{1}$, J. A. VILLETH ${ }^{1}$, F. A. BRAGGIO ${ }^{1}$, J. L. ZOTIN ${ }^{2}$, M. A. P. DA SILVA ${ }^{1}$ \\ ${ }^{1}$ Universidade Federal do Rio de Janeiro, Escola de Química \\ ${ }^{2}$ CENPES/ PETROBRAS \\ Email para contato: monica@eq.ufrj.br
}

\begin{abstract}
RESUMO - A preocupação quanto à emissão de poluentes, como o enxofre, em combustíveis fomenta a necessidade de desenvolvimento de processos de hidrotratamento mais eficazes. $O$ objetivo deste trabalho foi avaliar a hidrodessulfurização (HDS) de dibenzotiofeno (DBT) e 4,6-dimetildibenzotiofeno (4,6-DMDBT) em catalisadores $\mathrm{NiMoP} / \gamma \mathrm{Al}_{2} \mathrm{O}_{3}$ e sua inibição pela presença de quinolina. A metodologia de preparo dos catalisadores foi a técnica de impregnação ao ponto úmido, variando-se o teor de fósforo entre $0-4 \%(\mathrm{~m} / \mathrm{m})$ e mantendo-se fixos os teores de níquel e molibdênio. A composição química foi determinada por fluorescência de raios X. A técnica de quimissorção de NO foi utilizada para determinar a densidade de sítios ativos dos catalisadores. As reações foram realizadas em reator de leito gotejante. A distribuição de produtos indica que a HDS de DBT ocorre preferencialmente via rota de dessulfurização direta, enquanto a de 4,6-DMDBT via rota de hidrogenação. Os resultados indicam maiores conversões para o DBT e uma maior inibição da HDS de 4,6-DMDBT na presença de quinolina.
\end{abstract}

\section{INTRODUÇÃO}

Legislações cada vez mais restritivas quanto ao teor de poluentes emitidos para a atmosfera vêm impulsionando uma maior preocupação quanto às quantidades de enxofre emitidas, principalmente nos combustíveis derivados do petróleo, como o óleo diesel, responsável por cerca de $50 \%$ do consumo de energia no setor de transportes brasileiro. Desse modo, a retirada de enxofre através do processo de hidrodessulfurização (HDS) profunda torna-se fundamental, sendo necessário o uso de condições cada vez mais severas que exigem catalisadores mais ativos.

As reações de HDS ocorrem por duas rotas: dessulfurização direta (DDS) ou hidrogenólise, na qual ocorre a quebra de pelo menos uma ligação $\mathrm{C}-\mathrm{S}$ da molécula ou a hidrogenação de um dos anéis benzênicos (HID) levando à formação de intermediários hidrodibenzotiofênicos seguido da dessulfurização a ciclohexilbenzenos e biciclohexilbenzenos. A ocorrência preferencial de uma das rotas depende da forma como a molécula adsorve na superfície do sítio catalítico. Há evidências na literatura de que as moléculas de DBT reagem preferencialmente pela via DDS enquanto o 4,6-DMDBT reage 
preferencialmente pela via HID (Egorova e Prins, 2004; Stanislaus et al., 2010; GarcíaMartinez et al., 2012), o que ocorre provavelmente devido ao impedimento estérico do átomo de enxofre, causado pelos grupamentos metila.

Os catalisadores utilizados nas reações HDS são, geralmente, baseados em Ni (Co) e Mo (W) na forma de sulfetos mistos suportados em alumina. A adição de outros elementos, como o fósforo, vem sendo estudada na literatura (Sun et al., 2003).

As frações de petróleo apresentam não só compostos sulfurados, mas também compostos nitrogenados em sua composição, representando problema para o processo de HDS, uma vez que há uma competição entre os compostos nitrogenados e os sulfurados pela adsorção nos sítios ativos durante essas reações (García-Martinez et al., 2012). O impacto do efeito inibitório, no entanto, depende do tipo de composto nitrogenado presente no meio reacional. De acordo com Stanislaus et al. (2010), os compostos nitrogenados básicos como a quinolina são os que têm maiores efeitos sobre as reações de HDS.

O objetivo deste trabalho foi avaliar a hidrodessulfurização de DBT e 4,6-DMDBT na ausência e na presença de quinolina em catalisadores $\mathrm{NiMo} / \mathrm{Al}_{2} \mathrm{O}_{3}$, estudando seus efeitos de inibição. Desse modo, foram preparados três catalisadores com teores de fósforo variando entre 0 e $4 \% \mathrm{P}(\mathrm{m} / \mathrm{m})$, que foram caracterizados quanto à composição química e à densidade total de sítios ativos.

\section{PROCEDIMENTO EXPERIMENTAL}

\subsection{Preparo dos Catalisadores}

O preparo dos catalisadores foi realizado através da técnica de impregnação ao ponto úmido. A formulação adotada foi de $10 \%(\mathrm{~m} / \mathrm{m})$ de Mo e razão atômica $\mathrm{Ni} / \mathrm{Mo}=0,7$ (Morgado Jr. et al., 2009), com e sem a adição de fósforo (1 e $4 \%(\mathrm{~m} / \mathrm{m})$ ). Como precursores foram utilizados nitrato de níquel (P.A. Acros), heptamolibdato de amônio (P.A. Merck) e ácido fosfórico (85\% Isofar), sendo a alumina Pural SB (Sasol) na granulometria $+60-100$ mesh Tyler previamente seca usada como suporte e o $\mathrm{pH}$ da solução final ajustado em $3 \mathrm{com}$ ácido nítrico. Os catalisadores foram secos a $120^{\circ} \mathrm{C}$ por $12 \mathrm{~h}$, com calcinação posterior a $450{ }^{\circ} \mathrm{C}$ por $1 \mathrm{~h}$. A nomenclatura adotada foi NiMoxP, onde x é a porcentagem mássica de fósforo.

\subsection{Caracterização dos Catalisadores}

A análise de fluorescência de raios-X (FRX) dos catalisadores foi realizada através de um espectrômetro de raios X Rigaku modelo RIX 3100. A determinação dos sítios ativos totais foi realizada pela técnica de quimissorção de NO nos catalisadores sulfetados utilizando um equipamento AutoChem 2920 através de pulsos de uma mistura 10\% (v/v) NO/He. As amostras foram previamente tratadas in situ sob fluxo de ar sintético a $140{ }^{\circ} \mathrm{C}$ por 20 min, com posterior sulfetação utilizando uma mistura $1 \% \mathrm{H}_{2} \mathrm{~S} / \mathrm{H}_{2}$ a $400{ }^{\circ} \mathrm{C}\left(1{ }^{\circ} \mathrm{C} / \mathrm{min}\right)$ por $1 \mathrm{~h}$. 


\subsection{Testes Catalíticos}

A HDS de DBT (98\% Sigma-Aldrich) e de 4,6-DMDBT (97\% Sigma-Aldrich) foi realizada em reator de leito gotejante (PID Eng \& Tech). O reator foi carregado com aproximadamente $1,0 \mathrm{~g}$ de catalisador calcinado diluído com $1,5 \mathrm{~g}$ de $\mathrm{SiC}$, ambos na faixa de +60-100 mesh Tyler, de modo a minimizar escoamento preferencial e adequar às condições de escoamento bifásico. Para avaliar o progresso da reação, foi utilizada cromatografia gasosa, sendo o estado estacionário alcançado em $5 \mathrm{~h}$ de reação em média. A sulfetação foi realizada in situ utilizando uma solução $4 \%(\mathrm{~m} / \mathrm{m})$ de $\mathrm{CS}_{2}$ em n-hexano $(0,10 \mathrm{~mL} / \mathrm{min})$ a 31 bar de $\mathrm{H}_{2} \mathrm{e}$ $350{ }^{\circ} \mathrm{C}$ por $2 \mathrm{~h}$. Como carga reacional foi utilizada uma mistura de parafinas de $\mathrm{C} 13$ a $\mathrm{C} 18$ (CENPES) com $1000 \mathrm{mg} / \mathrm{kg}$ de enxofre para a avaliação da HDS, tanto de DBT quanto de 4,6-DMDBT. Já para avaliação do efeito de inibição da quinolina, foram utilizadas concentrações de 20, 120 e $300 \mathrm{mg} / \mathrm{kg}$ de nitrogênio, mantendo-se a mesma concentração de enxofre.

\section{RESULTADOS E DISCUSSÃO}

\subsection{Caracterização dos Catalisadores}

Na Tabela 1 são apresentadas a composição química e a densidade de sítios de ativos para os catalisadores NiMoxP calcinados.

Tabela 1 - Composição química e densidade de sítios ativos para os catalisadores calcinados

\begin{tabular}{|c|c|c|c|c|c|}
\hline \multirow{2}{*}{ Catalisador } & \multicolumn{4}{|c|}{$\begin{array}{c}\text { Composição química } \\
(\% \mathrm{~m} / \mathrm{m})\end{array}$} & \multirow{2}{*}{$\begin{array}{c}\text { Densidade de sítios } \\
\text { ativos }\left(\mu \mathrm{mols} \mathrm{NO} / \mathrm{g}_{\text {cat }}\right)\end{array}$} \\
\cline { 2 - 5 } & $\mathrm{Mo}$ & $\mathrm{Ni}$ & $\mathrm{P}$ & $\mathrm{Al}$ & \\
\hline NiMo0P & 9,8 & 2,9 & 0,0 & 43,2 & 150 \\
\hline NiMo1P & 9,1 & 2,8 & 1,3 & 42,4 & 165 \\
\hline NiMo4P & 9,1 & 2,7 & 4,1 & 38,9 & 113 \\
\hline
\end{tabular}

Analisando a Tabela 1, observa-se que os teores reais são similares aos nominais. A adição de $1 \%(\mathrm{~m} / \mathrm{m})$ de $\mathrm{P}$ promove um aumento na densidade de sítios ativos dos catalisadores, corroborando a literatura (Sun et al., 2003), no entanto para o teor de $4 \%(\mathrm{~m} / \mathrm{m})$ de $\mathrm{P}$ observou-se uma diminuição da densidade total de sítios ativos.

\subsection{Testes Catalíticos}

Com base no resultado da quimissorção, o catalisador NiMo1P foi empregado para avaliar a HDS de DBT e de 4,6-DMDBT na ausência e presença de quinolina. As condições experimentais e resultados obtidos para HDS na ausência de nitrogenados estão apresentados nas Tabelas 2 e 3. Para avaliar o efeito da inibição por quinolina foi utilizada a condição de $275^{\circ} \mathrm{C}, 31$ bar e WHSV de $4 \mathrm{~h}^{-1}$ para o 4,6-DMDBT e $16 \mathrm{~h}^{-1}$ para o DBT. Para as reações de HDS de DBT, foram formados somente bifenil (BF) e ciclohexilbenzeno (CHB) e para as reações com 4,6-DMDBT foram formados 3,3-dimetilbifenil (3,3-DMBF), metilciclohexiltolueno (MCHT) e dimetilbiciclohexano (DMBCH). 
Tabela 2 - Condições experimentais utilizadas na HDS

\begin{tabular}{|c|c|c|c|}
\hline \multirow{2}{*}{ Condição } & \multicolumn{3}{|c|}{ Condições } \\
\cline { 2 - 4 } & $\begin{array}{c}\text { Temperatura } \\
\left({ }^{\circ} \mathrm{C}\right)\end{array}$ & $\begin{array}{c}\text { Pressão } \\
(\text { bar })\end{array}$ & $\begin{array}{c}\text { WHSV } \\
\left(\mathrm{h}^{-1}\right)\end{array}$ \\
\hline 1 & 230 & 31 & 4 \\
\hline 2 & 230 & 51 & 8 \\
\hline 3 & 245 & 31 & 4 \\
\hline 4 & 245 & 31 & 8 \\
\hline
\end{tabular}

Tabela 3 - Resultados da HDS na ausência de quinolina

\begin{tabular}{|c|c|c|c|c|c|c|c|}
\hline \multirow{3}{*}{ Condição } & \multicolumn{3}{|c|}{ DBT } & \multicolumn{4}{|c|}{ 4,6-DMDBT } \\
\hline & \multirow{2}{*}{$\begin{array}{c}\text { Conversão } \\
\text { DBT (\%) }\end{array}$} & \multicolumn{2}{|c|}{$\begin{array}{c}\text { Rendimento } \\
(\%)\end{array}$} & \multirow{2}{*}{$\begin{array}{c}\text { Conversão } \\
4,6- \\
\text { DMDBT } \\
(\%)\end{array}$} & \multicolumn{3}{|c|}{ Rendimento (\%) } \\
\hline & & $\mathrm{BF}$ & $\mathrm{CHB}$ & & $\begin{array}{c}3,3- \\
\text { DMBF }\end{array}$ & MCHT & $\mathrm{DMBCH}$ \\
\hline 1 & 70,9 & 54,2 & 18,2 & 26,9 & 0,9 & 6,0 & 5,4 \\
\hline 2 & 53,4 & 37,3 & 13,8 & 16,8 & 0,3 & 3,5 & 2,7 \\
\hline 3 & 96,5 & 69,3 & 27,8 & 40,6 & 1,9 & 13,1 & 13,8 \\
\hline 4 & 78,8 & 61,8 & 17,1 & 25,6 & 1,4 & 6,4 & 5,3 \\
\hline
\end{tabular}

De acordo com a Tabela 3, a reação de HDS de DBT ocorreu majoritariamente através da rota DDS, uma vez que os rendimentos de BF foram superiores aos de CHB. No entanto, na HDS de 4,6-DMDBT a rota preferencial foi a HID. Percebe-se também que, para condições similares, as conversões de DBT são muito maiores do que as de 4,6-DMDBT, corroborando a literatura (Stanislaus et al., 2010; García-Martinez et al., 2012). Na Tabela 4 estão apresentados os resultados das reações de HDS na presença de quinolina.

Tabela 4 - Resultados da HDS na presença de quinolina

\begin{tabular}{|c|c|c|c|c|c|c|c|}
\hline \multirow{3}{*}{$\begin{array}{l}\text { Conc. N } \\
\text { (mg/kg) }\end{array}$} & \multicolumn{3}{|c|}{ DBT } & \multicolumn{4}{|c|}{ 4,6-DMDBT } \\
\hline & \multirow{2}{*}{$\begin{array}{c}\text { Conversão } \\
\text { DBT }(\%)\end{array}$} & \multicolumn{2}{|c|}{$\begin{array}{c}\text { Rendimento } \\
(\%)\end{array}$} & \multirow{2}{*}{$\begin{array}{c}\text { Conversão } \\
4,6- \\
\text { DMDBT } \\
(\%)\end{array}$} & \multicolumn{3}{|c|}{ Rendimento (\%) } \\
\hline & & $\mathrm{BF}$ & $\mathrm{CHB}$ & & 3,3-DMBF & MCHT & $\mathrm{DMBCH}$ \\
\hline $0_{\text {inicial }}$ & 95,5 & 90,8 & 4,3 & 83,0 & 5,4 & 19,8 & 32,7 \\
\hline 20 & 93,9 & 89,1 & 2,9 & 67,6 & 4,7 & 20,3 & 26,3 \\
\hline 120 & 85,2 & 85,2 & 0,9 & 14,0 & 1,4 & 2,4 & 1,8 \\
\hline 300 & 76,2 & 76,5 & 0,4 & 3,3 & 0,8 & 0,4 & 0,5 \\
\hline
\end{tabular}

A partir da Tabela 4, percebe-se que o aumento da concentração de quinolina leva a diminuição da conversão, tanto de DBT quanto de 4,6-DMDBT. Houve alteração na distribuição de produtos das reações, para teores mais elevados de quinolina (120 ppmN), sendo a rota HID a mais inibida. Desse modo, observou-se maior redução dos rendimentos em CHB, MCHT e DMBCH em relação ao BF e 3,3-DMBF (Stanislaus et al., 2010; Egorova e Prins, 2004). Através de estudo realizado anteriormente (Polck, 2010), sabe-se que a atividade 
de HDS de DBT é regenerada totalmente após a retirada da quinolina do meio reacional, enquanto a de 4,6-DMDBT não.

A inibição da HDS pela quinolina foi avaliada através da razão entre as atividades de HDS na presença de quinolina e na ausência de quinolina (Figura 1). Para possibilitar a comparação entre reações realizadas na mesma temperatura e pressão e com WHSV distintos, foi realizada uma extrapolação considerando a reação global como de pseudo-primeira ordem e a constante de reação dependente somente da temperatura.

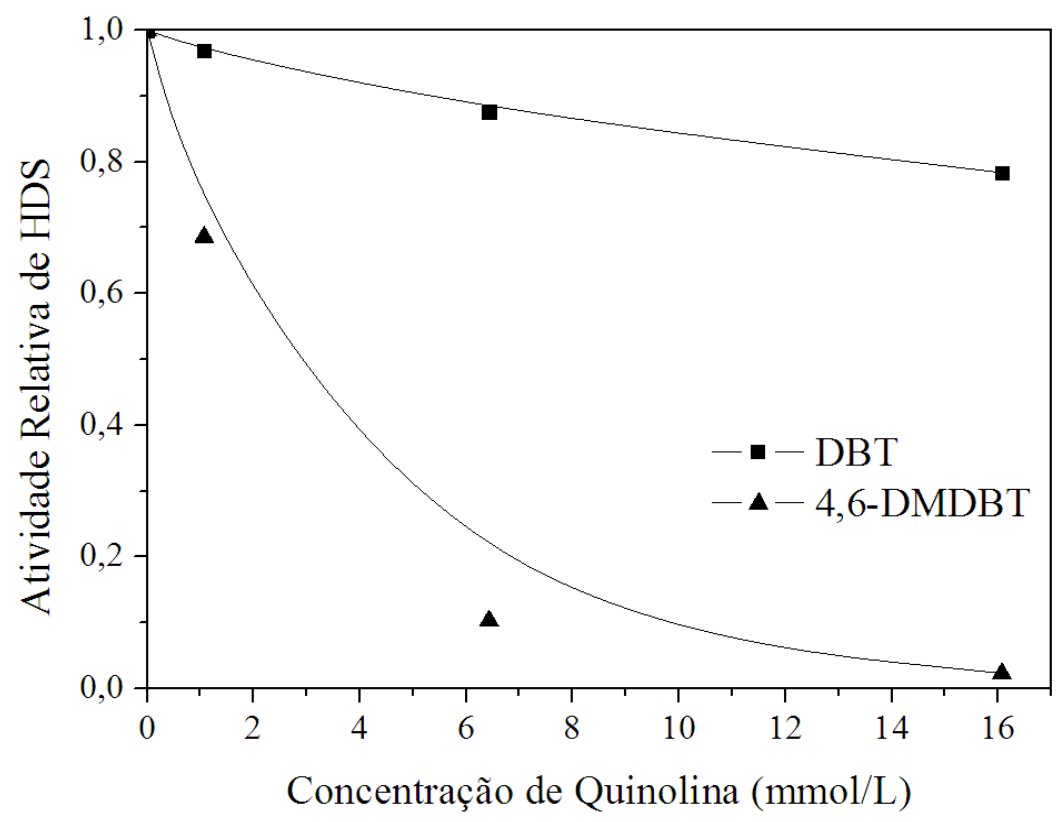

Figura 1 - Atividade relativa em função da concentração inicial de quinolina $\left(275^{\circ} \mathrm{C}, 31\right.$ bar e $\left.16 \mathrm{~h}^{-1}\right)$.

A partir da Figura 1, percebe-se maior efeito inibitório da quinolina na HDS de 4,6-DMDBT, visto que para baixas concentrações de quinolina ( $1 \mathrm{mmol} / \mathrm{L})$ há uma diminuição de $30 \%$ na atividade , enquanto para o DBT a maior redução da atividade foi de $20 \%$ para a maior concentração de nitrogenado avaliada $(16 \mathrm{mmol} / \mathrm{L})$. Esse comportamento já foi observado na literatura (Kun e Flora, 2010).

Quanto à realização de HDN, percebeu-se que toda a quinolina foi convertida. Contudo, houve a formação de intermediários nitrogenados, sendo, desse modo, a conversão em produtos de HDN inferior a de quinolina. Na Figura 2 estão apresentadas as conversões de HDN em função das concentrações iniciais de quinolina para as duas cargas sulfuradas, segundo metodologia apresentada por Infantes-Molina et al. (2012).

Pela Figura 2, percebe-se que houve redução da conversão de HDN com o aumento da concentração de quinolina, tendo comportamento linear para a carga com 4,6-DMDBT. Através da comparação das Figuras 1 e 2, nota-se que para os ensaios com 4,6-DMDBT há predomínio da HDN, fato também observado por Turaga et al.(2003). Já para reações com 
DBT, a HDN é mais afetada pela presença do composto sulfurado do que a HDS pela da quinolina (Egorova e Prins, 2004).

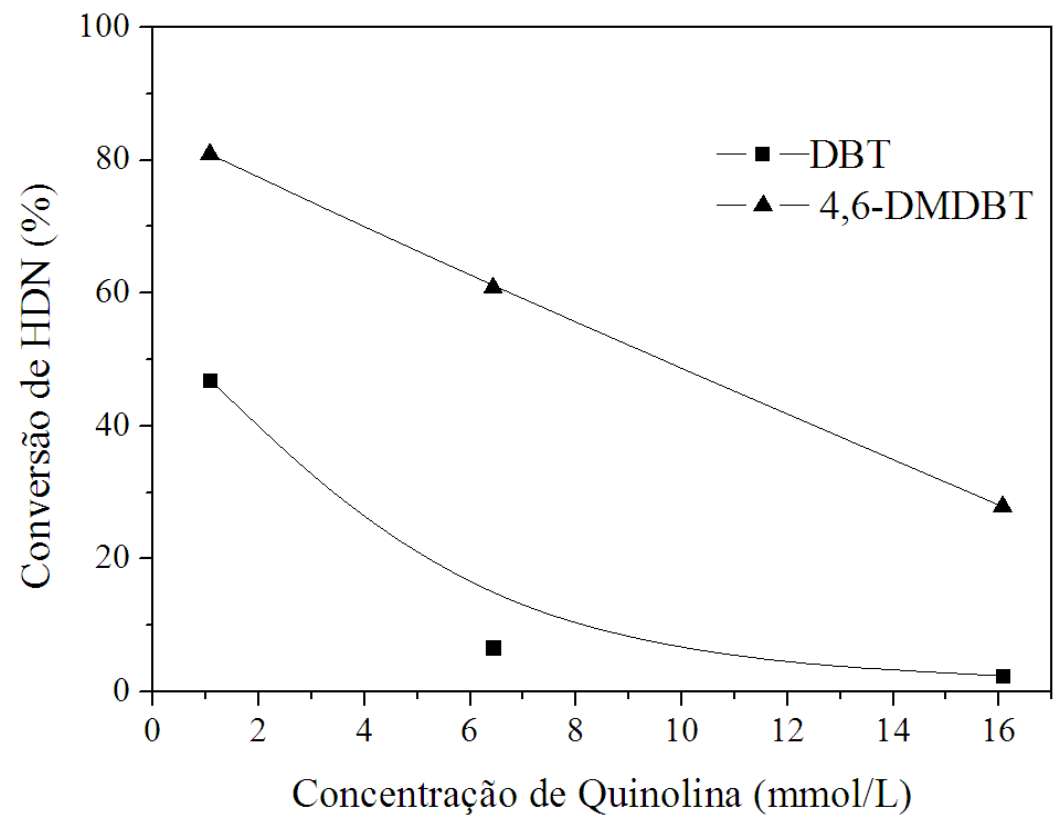

Figura 2 - Conversão de HDN vs concentração inicial de quinolina.

Outra forma de representar a inibição das reações de HDS na presença de compostos nitrogenados é a obtenção da constante de equilíbrio de adsorção. Laredo et al. (2001) propuseram um modelo baseado em Langmuir-Hinshelwood para determinar a constante de equilíbrio de adsorção da quinolina, representado na Equação 1.

$$
r_{H D S}=\frac{k_{S U L F} C_{S U L F}}{1+\left(K_{N} C_{Q U I N 0}\right)^{n}}
$$

onde: $\mathrm{k}_{\mathrm{SULF}}$ constante da taxa intrínseca $\left(\mathrm{h}^{-1}\right) ; \mathrm{K}_{\mathrm{N}}$ : constante de equilíbrio de adsorção aparente do composto nitrogenado (L/mmol); C C SULF: concentração de DBT ou de 4,6DMDBT (mmol/L); $C_{\text {Quino: }}$ concentração inicial de quinolina (mmol/L); e n: ordem parcial de reação em função da quinolina.

A taxa de reação na presença de quinolina pode ser escrita conforme apresentado na Equação 2, segundo metodologia de Laredo et al. (2001).

$$
r_{H D S}=k_{S U L F}^{\prime} C_{S U L F}
$$

onde: k'sulF: constante da taxa de reação de pseudo-primeira ordem da HDS de DBT ou 4,6-DMDBT, com influência de quinolina.

Substituindo a Equação 2 na Equação 1:

$$
k_{S U L F}^{\prime}=\frac{k_{S U L F}}{1+\left(K_{N} C_{Q U I N 0}\right)^{n}}
$$


Por meio do rearranjo da Equação 3, obtém-se $\mathrm{K}_{\mathrm{N}}$ a partir do valor do expoente ajustável n (Tabela 5).

Tabela 5 - Constantes de equilíbrio de adsorção aparente para diferentes valores de $\mathrm{n}$

\begin{tabular}{|c|c|c|c|}
\hline \multirow{2}{*}{$\begin{array}{c}\text { Composto } \\
\text { modelo }\end{array}$} & $\mathrm{n}$ & $\begin{array}{c}\text { Constante de Equilíbrio } \\
\text { de Adsorção Aparente } \\
\mathrm{K}_{\mathrm{N}}(\mathrm{L} / \mathrm{mol})\end{array}$ & $\begin{array}{c}\text { Coeficiente de } \\
\text { Determinação } \\
\left(\mathrm{R}^{2}\right)\end{array}$ \\
\cline { 1 - 2 } DBT & \multirow{2}{*}{1} & 95 & 0,95 \\
\cline { 1 - 1 } 4,6-DMDBT & 2954 & 0,93 \\
\hline yBT & \multirow{2}{*}{0,5} & 11 & 0,99 \\
\cline { 1 - 3 } 4,6-DMDBT & 311 & 0,74 \\
\hline
\end{tabular}

A partir da Tabela 5, observa-se melhor ajuste para a reação com 4,6-DMDBT para $\mathrm{n}=1$ e para $n=0,5$ para a reação com DBT. A constante de equilíbrio de adsorção para o catalisador NiMo1P com 4,6-DMDBT foi muito superior à obtida com DBT para todos os ajustes avaliados, indicando maior inibição da quinolina na HDS do 4,6-DMDBT como mostrado anteriormente na Figura 1 e relatado por Kun e Flora (2010). A boa adequação da equação cinética adotada utilizando a concentração inicial de quinolina sugere que a inibição seja constante no decorrer da reação.

Sugere-se que a grande diferença nas constantes de adsorção da quinolina em reações com DBT e 4,6-DMDBT esteja associada à natureza dos sítios envolvidos em cada reação. De acordo com a distribuição de produtos mostrada anteriormente, há uma queda muito acentuada na formação de produtos de hidrogenação, para ambas as moléculas. Assim, a quinolina se adsorve preferencialmente nestes sítios e, uma vez que o 4,6-DMDBT é convertido majoritariamente pela rota hidrogenação, o efeito líquido de inibição é muito mais pronunciado para esta molécula que para o DBT. Foram observados valores na mesma ordem de grandeza para trabalhos envolvendo os compostos modelos (Laredo et al., 2004; Polck, 2010; García-Martinez et al., 2012).

\section{CONCLUSÕES}

A quimissorção de NO indicou que o catalisador NiMo1P possui maior densidade de sítios ativos totais. As reações de HDS mostram que o 4,6-DMDBT é mais refratário que o DBT. Na HDS de DBT, observou-se predominância da rota DDS em detrimento da HID, enquanto o 4,6-DMDBT reagiu preferencialmente via rota HID. Quanto ao efeito da quinolina na HDS, as reações com 4,6-DMDBT sofrem inibição mais significativa e a rota HID a mais inibida para ambos os compostos. As constantes de equilíbrio de adsorção obtidas para quinolina indicam predominância da HDN face à HDS para o 4,6-DMDBT, efeito oposto ao do DBT.

\section{AGRADECIMENTOS}

À PETROBRAS S.A. pelo apoio financeiro através do convênio 0050.0071477.11.9. À ANP, à FINEP, ao CNPq e ao PRH-13 pelo apoio financeiro aos alunos Matheus Dorneles de Mello e Flávia de Almeida Braggio. Ao NUCAT (PEQ/COPPE/UFRJ) pelas análises de FRX. 


\section{REFERÊNCIAS}

EGOROVA, M.; PRINS, R. Mutual influence of the HDS of dibenzothiophene and HDN of 2-methylpyridine. J. Catal., v.221, p. 11-19, 2004.

GARCÍA-MARTINEZ, J.C.; CASTILLO-ARAIZA, C.O.; HEREDIA, J.A.R.; TREJO, E.; MONTESINOS, A. Kinetics of HDS and of the inhibitory effect of quinolina on HDS of 4,6-DMDBT over a Ni-Mo-P/ $\mathrm{Al}_{2} \mathrm{O}_{3}$ catalyst: Part I. Chem. Eng. J., v. 210, p. 53-62, 2012.

INFANTES-MOLINA， A.; MORENO-LEÓN， C.; PAWELEC， B.; FIERRO， J.L.G.; RODRÍGUES-CASTELLÓN, E. Simultaneous hydrodesulfurization and hydrodenitrogenation on $\mathrm{MoP} / \mathrm{SiO}_{2}$ catalysts: Effect of catalyst preparation method. Appl. Catal. B, v. 113, p. 87-99, 2012.

KUN, L.; FLORA, T.T.N.G. Effect of the nitrogen heterocyclic compounds on hydrodesulfurization using in situ hydrogen and a dispersed Mo catalyst. Catal. Today, v. 149, p.28-34, 2010.

LAREDO, G.C.; DE LOS REYES, J. A.; CANO, J. L.; CASTILLO, J. J. Inhibition effects of nitrogen compounds on the hydrodesulfurization of dibenzothiophene. Appl. Catal. A, v. 207, p. 103-112, 2001.

LAREDO, G. C.; MONTESINOS, A.; DE LOS REYES, J. A. Inhibition effects observed between dibenzothiophene and carbazole during the hydrotreating process. Appl. Catal. A, v. 265, p. 171-183, 2004.

MORGADO JR, E.; ZOTIN, J.L.; ABREU, M.A.S.; ROSAS, D.O.; JARDIM, P.M.; MARINKOVIC, B.A. Characterization and hydrotreating performance of NiMo catalysts supported on nanostructured titanate. Appl. Catal. A, v. 357, p. 142-147, 2009.

POLCK, D. Estudo cinético da reação de hidrodessulfurização de moléculas modelo de dibenzotiofeno e 4,6-dimetidibenzotiofeno e efeitos de inibição de compostos nitrogenados, Dissertação de Mestrado, EQ/UFRJ, Rio de Janeiro, 2010.

STANISLAUS, A.; MARAFI, A.; RANA, M.S. Recent advances in the science and technology of ultra low sulfur diesel (ULSD) production. Catal. Today, v. 153, p. 1- 68, 2010 .

SUN, M., NICOSIA, D.; PRINS, R. The effects of fluorine, phosphate and chelating agents on hydrotreating catalysts and catalysis. Catal. Today, v. 86, p. 173-189, 2003.

TURAGA, U.T.; MA, X.; SONG, C. Influence of nitrogen compounds on deep hydrodesulfurization of 4,6-dimethyldibenzothiophene over $\mathrm{Al}_{2} \mathrm{O}_{3}$ - and MCM-41supported Co-Mo sulfide catalysts. Catal. Today, v.86, p. 265-275, 2003. 